\title{
Saturday night purpura: An uncommon presentation of exercise-induced vasculitis
}

\begin{abstract}
Exercise-induced vasculitis (EIV) is a benign cutaneous vasculitis that affects healthy individuals. We report a case of EIV in a 15-year-old male patient with a striking clinical appearance triggered by a combination of prolonged standing and disco dancing in a warm environment. Pediatric dermatologists should be aware of this activity as a possible trigger of EIV, especially in teenagers.
\end{abstract}

\section{1 | INTRODUCTION}

Exercise-induced vasculitis (EIV), also known as golfer's vasculitis, is a benign cutaneous disorder that affects healthy individuals. ${ }^{1}$ The disorder is typically triggered by prolonged or excessive exercise in warm weather. Lesions appear as erythematous, urticarial, or purpuric plaques symmetrically distributed on the lower extremities, sparing areas of compression (eg, by socks), and it is not associated with any systemic symptoms. ${ }^{2-5}$ EIV appears most commonly in women over the age of 50 years and it has rarely been reported in children; only six cases have been published. ${ }^{2,5,6}$ We present a case of EIV in an adolescent male that appeared after a night of dancing.

\section{2 | CASE REPORT}

A 15-year-old male presented to the Emergency Department with a 3-year history of recurrent outbreaks of itchy purpuric lesions on his lower limbs. Patient reported that the skin lesions appeared only after weekend trips to the disco, associated with prolonged standing and dancing. The lesions appeared predominantly in the summer months. Spontaneous resolution occurred within a few days without treatment. No extracutaneous manifestations were reported. Clinical examination revealed purpuric macules, coalescing into symmetrical patches on both sides of the legs and thighs, with relative sparing of the feet (Figure 1).

Cutaneous biopsy demonstrated a vasculitic process with leukocytoclasia and a perivascular infiltrate composed predominantly of neutrophils in superficial dermis (Figure 2). At higher magnification, bulging endothelial cells, red blood cell extravasation, and karyorrhexis were observed. Perls's iron stain was

negative. Direct immunofluorescence (DIF) did not reveal complement or immunoglobulin deposits. Standard and immunological blood tests including complete blood count, renal and hepatic function, autoimmune antibodies (ANA, ANCA, dsDNA, and ENA), circulating immune complexes, electrophoresis of plasma proteins, complement levels (C3 and C4), rheumatoid factor, erythrocyte sedimentation rate, C-reactive protein, urinalysis, serological tests for syphilis, group A Streptococcus, human immunodeficiency virus, and hepatitis $B$ and $C$ were negative or within normal limits.

Given the clinical presentation, associated trigger and complementary test results, a diagnosis of exercise-induced vasculitis was made. The patient was treated with compression stockings during activity, resulting in a moderate reduction in the development of skin lesions during the subsequent 6 months of follow-up.

\section{3 | DISCUSSION}

The pathogenesis of EIV remains uncertain and is likely multifactorial. $^{3}$ Possible contributory factors include excessive heat production during vigorous exercise leading to muscle hyperthermia and tissue damage. In addition, alterations of the immune system (with an increase in circulating immune complexes and complement activation) increase muscular arterial flow, and greater capillary permeability, causing petechiae and vasculitis. ${ }^{2,4}$

According to Espitia et al, ${ }^{5}$ leukocytoclastic vasculitis is the most common histological finding in EIV, with a neutrophilic perivascular infiltrate, nuclear dust, erythrocyte extravasation and swelling of the vessel walls. Deposits of C3, C1q and IgM in DIF and fibrinoid necrosis may sometimes be found, but are not constant findings. ${ }^{2,5}$ Blood count, complement, plasma proteins electrophoresis, antineutrophil cytoplasmic antibodies, antinuclear antibodies (ANA), extractable nuclear antigen antibodies (ENA), anti-double stranded DNA antibodies (anti-dsDNA), cryoglobulins, cold agglutinins, erythrocyte sedimentation rate, C-reactive protein, rheumatoid factor and urinalysis are all negative or within normal limits. ${ }^{5}$

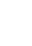

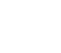



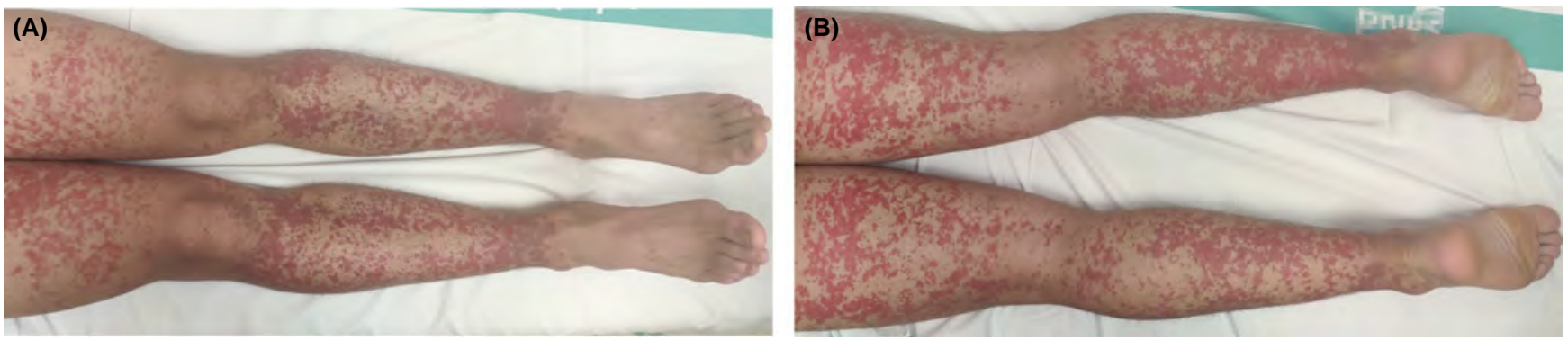

FIGURE 1 A, B, Purpuric macules coalescing in symmetric patches on the legs and thighs. Note transition line on the ankles with feet largely spared
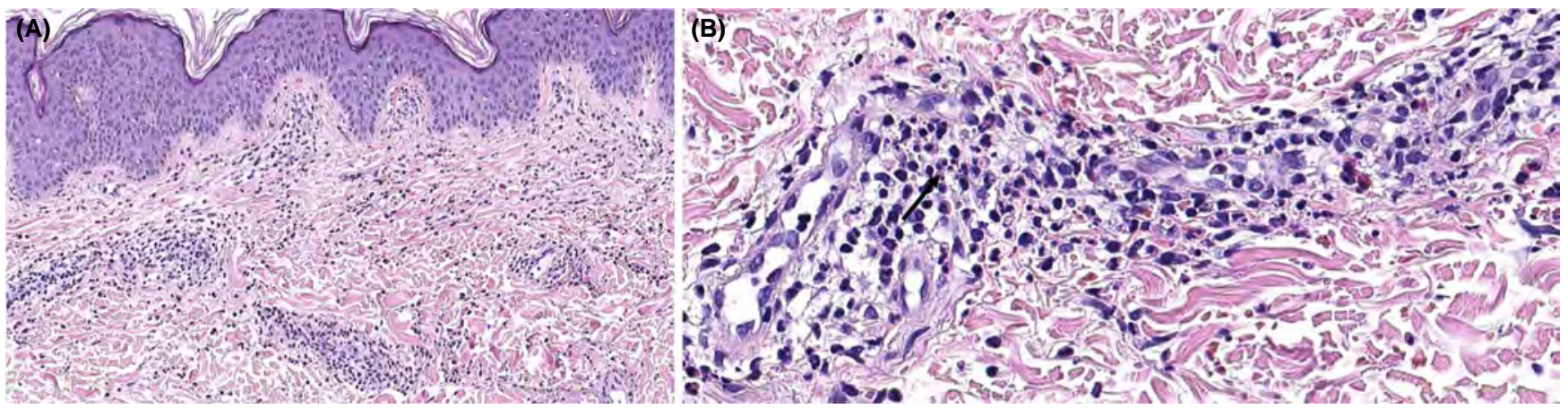

FIGURE 2 A, Normal epidermis with perivascular inflammatory infiltrates in the superficial dermis. Erythrocyte extravasation is observed without fibrinoid necrosis. (H\&E, $\times 20)$. B, Detail of a vessel showing bulged endothelial cells and an infiltration composed predominantly of neutrophils, with fewer eosinophils, and associated karyorrhexis (arrow) (H\&E, $\times 40$ )

involvement and IgA perivascular deposits. Systemic vasculitis is accompanied by organ involvement and laboratory abnormalities. ${ }^{2}$ Exercise-induced capillaritis should also be considered in the differential diagnosis, which shows a lymphohistiocytic perivascular infiltrate of small-caliber vessels in the superficial dermis, with endothelial cell edema and erythrocyte extravasation. In addition, Perls's stain demonstrates the presence of iron (hemosiderin) in the superficial dermis. Unlike EIV, leukocytoclasia is not observed in exercise-induced capillaritis. ${ }^{7,8}$

Although some authors have suggested that EIV is a form of exercise-induced capillaritis exacerbated by underlying subclinical venous hypertension, ${ }^{9}$ most authors classify it as a benign cutaneous variant of leukocytoclastic vasculitis. ${ }^{2-5}$ In fact, leukocytoclastic vasculitis is the main histopathological finding and neutrophils, with associated karyorrhexis, predominate in the perivascular infiltrate. ${ }^{2,5}$ Since EIV is considered a benign vasculopathy, in the absence of a primary inflammatory vasculitis, some authors prefer the term exercise-induced purpura. ${ }^{2}$

Exercise-induced vasculitis usually resolves spontaneously in a few days, although relapses are frequent. ${ }^{5}$ Treatment is not standardized, and prognosis is good given the self-resolving nature of lesions. Nevertheless, topical corticosteroids can be used for associated pruritus. Wearing light clothing, avoiding sports in warm weather, and use of compression stockings during activity may protect against EIV. This last intervention was partially helpful in our patient, with a reduction in the severity of outbreaks.
It is important to recognize EIV in order to provide reassurance to patients and their family regarding the benign nature of the eruption. However, given the histological finding of leukocytoclastic vasculitis, EIV should be a diagnosis of exclusion and systemic involvement should always be excluded.

\section{KEYWORDS}

cutaneous, diagnosis, exercise, leukocytoclastic, vasculitis

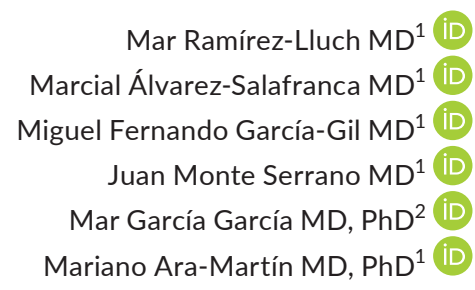

${ }^{1}$ Department of Dermatology, Hospital Clínico Universitario Lozano Blesa, Zaragoza, Spain

${ }^{2}$ Department of Pathology, Hospital Clínico Universitario Lozano

Blesa, Zaragoza, Spain

\section{Correspondence}

Mar Ramírez Lluch, MD, Department of Dermatology, Hospital Clínico Universitario Lozano Blesa, Avda San Juan Bosco, 15, 50009, Zaragoza, Spain. Email: mar.ramirezlluch@gmail.com 


\section{ORCID}

Mar Ramírez-Lluch iD https://orcid.org/0000-0003-0267-5901

Marcial Álvarez-Salafranca iD https://orcid.

org/0000-0002-1691-3280

Miguel Fernando García-Gil iD https://orcid.

org/0000-0002-2807-2730

Juan Monte Serrano iD https://orcid.org/0000-0002-0439-1200

Mar García García iD https://orcid.org/0000-0003-2078-8205

Mariano Ara-Martín iD https://orcid.org/0000-0001-8789-6783

\section{REFERENCES}

1. Kelly RI, Opie J, Nixon R. Golfer's vasculitis. Australas J Dermatol. 2005;46:11-14.

2. Paul SS, Scalzi LV. Exercise-induced purpura in children. Pediatrics. 2019;143(4):e20182797.
3. Li W, Moran A, Kim ESH. Golfer's vasculitis. Vasc Med. 2019;24(3):265-266.

4. Jud P, Hafner F. Exercise-induced vasculitis. CMAJ. 2018;190(7):E195.

5. Espitia O, Dréno B, Cassagnau E, et al. Exercise-induced vasculitis: a review with illustrated cases. Am J Clin Dermatol. 2016;17(6): 635-642.

6. Leung AK, Grant RM, Truscott R. Exercise-induced purpura. J Sports Med Phys Fitness. 1990;30(3):329-330.

7. Martínez Pallás I, Conejero del Mazo R, Lezcano BV. Pigmented purpuric dermatosis: a review of the literature. Actas Dermosifiliogr. 2020;111(3):196-204.

8. Hwa C, Brauer JA, Mundi JP, et al. Exercise-induced progressive pigmentary purpura of the forehead. J Am Acad Dermatol. 2011;65(5):e1 49-e150.

9. Sanyal S, Tsang Y, Miller J, Ilchyshyn A. Golfer's purpura - an under recognised form of exercise-induced capillaritis. J Eur Acad Dermatol Venereol. 2016;30(8):1403-1404. 